\title{
Plasticity of NMDA Receptors at Ventral Hippocampal Synapses in the Infralimbic Cortex Regulates Cued Fear
}

\author{
DiD Omar Soler-Cedeño, ${ }^{1}$ Orlando Torres-Rodríguez, ${ }^{1}$ Faviola Bernard, ${ }^{2}$ Lizette Maldonado, ${ }^{1}$ Anixa \\ Hernández, ${ }^{1}$ and James T. Porter ${ }^{1}$
}

https://doi.org/10.1523/ENEURO.0354-18.2019

${ }^{1}$ Department of Basic Sciences, Ponce Research Institute, Ponce Health Sciences University, Ponce, PR 00732 and ${ }^{2}$ Department of Biology, University of Puerto Rico-Ponce, Ponce, PR 00734

\section{Visual Abstract}

\section{NMDAR Currents at Ventral Hippocampal Synapses in the Infralimbic Cortex Vary with Fear Levels}

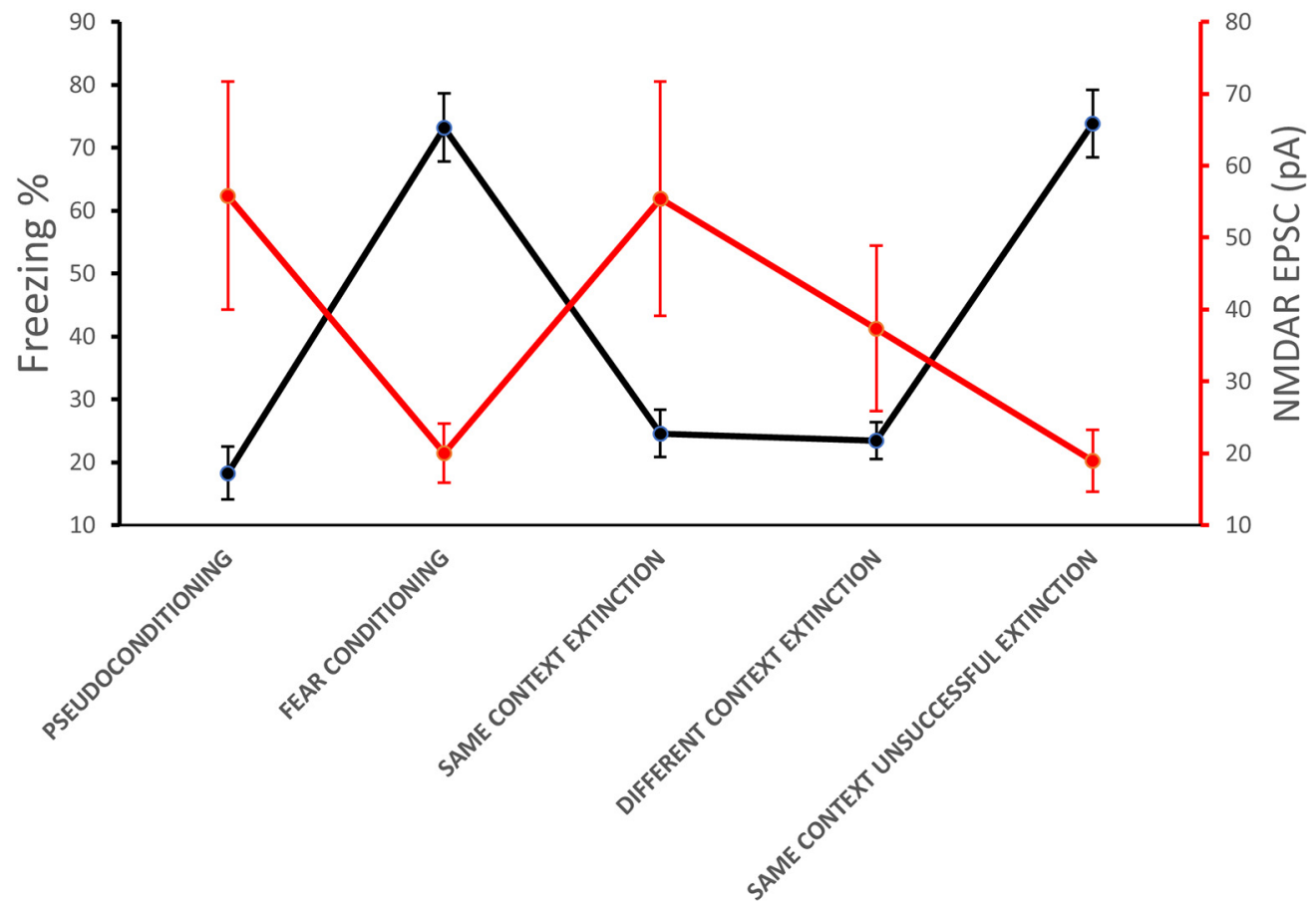

The medial prefrontal cortex (mPFC) processes contextual information from the hippocampus to generate appropriate fear responses. In rodents, one path for sending contextual information to the mPFC is via the direct projections from the ventral hippocampus $(\mathrm{vHC})$ to the infralimbic cortex (IL). Plasticity in the synaptic communication from the $\mathrm{VHC}$ to the IL could contribute to the behavioral changes produced by the acquisition and extinction of conditioned fear. To examine this possibility, we used optogenetic stimulation of vHC synapses in brain slices from trained rats. We found that fear acquisition reduced NMDA receptor (NMDAR) currents at vHC synapses onto IL pyramidal neurons. The depression of NMDAR currents reversed more efficiently after extinction in the conditioning context than extinction in a novel context. Moreover, a cohort of animals that exhibited poor extinction retrieval failed to reverse the plasticity induced by fear conditioning. In addition, ex vivo application of 
brain-derived neurotrophic factor (BDNF), which is known to simulate extinction in IL, reversed this conditioninginduced plasticity mimicking extinction. Therefore, we have identified a novel mechanism that modulates conditioned fear via changes in NMDAR current at VHC synapses onto IL pyramidal neurons. Disruption of this mechanism could contribute to the abnormal contextual modulation of fear seen in posttraumatic stress disorder (PTSD).

Key words: fear conditioning; fear extinction; NMDA receptor; prefrontal cortex; PTSD; ventral hippocampus

\section{Significance Statement}

Contextual information processing by the medial prefrontal cortex (mPFC) is critical for appropriate behavioral responses. Using optogenetics in brain slices, we found that acquisition of conditioned fear depressed NMDAR currents at ventral hippocampal synapses in the infralimbic cortex (IL) and extinction reversed the depression. Brain-derived neurotrophic factor (BDNF) could also reverse the conditioninginduced depression mimicking extinction. In contrast, animals with impaired fear extinction recall, a posttraumatic stress disorder (PTSD)-like phenotype, failed to reverse the conditioning-induced changes. Our findings suggest that conditioned fear responses are modulated by changes in NMDAR current at ventral hippocampal synapses in IL and suggest a novel mechanism that could contribute to impaired contextual modulation of fear seen in patients with PTSD.

\section{Introduction}

Contextual information is crucial to the generation of appropriate responses to environmental cues. Inappropriate contextual information contributes to various psychopathological conditions. For example, patients with PTSD show deficits in discriminating safe and dangerous contexts (RougemontBücking et al., 2011; Garfinkel et al., 2014). Functional imaging studies show that patients with PTSD have abnormal activity in the ventromedial prefrontal cortex (vmPFC), amygdala, and hippocampus during Pavlovian fear conditioning and extinction (Milad et al., 2009; Rougemont-Bücking et al., 2011). These brain structures are closely implicated in contextual processing (Maren et al., 2013) suggesting that deficits in activation of these structures likely contribute to the discrimination deficits seen in these patients (Garfinkel et al., 2014).

Fear extinction is context-dependent (Bouton and Bolles, 1979; Bouton, 2004; Kalisch et al., 2006; Langton and Richardson, 2009) and involves an interconnected neural circuit composed of the mPFC, hippocampus, and amygdala (Kalisch et al., 2006; Milad et al., 2007). The ventral hippocampus (vHC) conveys contextual information and synaptically excites neurons in the infralimbic

Received September 7, 2018; accepted February 10, 2019; First published March 14, 2019.

The authors declare no competing financial interests.

Author contributions: O.S.-C., O.T.-R., and J.T.P. designed research; O.S.-C., O.T.-R., F.B., L.M., and A.H. performed research; O.S.-C., O.T.-R., F.B., L.M., A.H., and J.T.P. analyzed data; O.S.-C., O.T.-R., and J.T.P. wrote the paper.

This work was supported by the Research Centers in Minority Institutions BRAIN and MAGIC Cores Grant NIMHHD G12 MD007579-27, the Ponce Health Sciences University and University of Puerto Rico-Ponce Research Initiative for Scientific Enhancement Programs R25 GM082406 and R25 GM096955, and National Institutes of Health Grants R15 MH101700 and F31 MH106288.

Correspondence should be addressed to James T. Porter at jporter@psm.edu

https://doi.org/10.1523/ENEURO.0354-18.2019

Copyright $\odot 2019$ Soler-Cedeño et al.

This is an open-access article distributed under the terms of the Creative Commons Attribution 4.0 International license, which permits unrestricted use, distribution and reproduction in any medium provided that the original work is properly attributed. cortex (IL); Maren et al., 2013; Kim and Cho, 2017, the homologous region to the vmPFC in rodents. Since the stimulation of IL pyramidal neurons inhibits conditioned fear (Milad et al., 2004; Bukalo et al., 2015; Do-Monte et al., 2015), synaptic communication between the vHC and IL could mediate the context-dependency of extinction (Sierra-Mercado et al., 2011; Orsini et al., 2013; Kim and Cho, 2017; Marek et al., 2018). However, whether $\mathrm{VHC}$ synapses in IL are altered by fear conditioning or extinction remains unknown.

In this study, we investigated whether fear conditioning or extinction alter the synaptic efficacy of $\mathrm{vHC}$ synapses onto IL pyramidal neurons. Additionally, we examined whether extinction given in the conditioning context induces similar synaptic plasticity as extinction in a novel context. To address these possibilities, we combined whole-cell patchclamp electrophysiology and optogenetics in brain slices with Pavlovian fear conditioning and extinction to specifically examine $\mathrm{vHC}$ synapses onto IL pyramidal neurons. In contrast to previous studies that did not find any plasticity of globally-activated synapses in IL after fear conditioning (Pattwell et al., 2012; Sepulveda-Orengo et al., 2013), we found that fear conditioning induced plasticity in $\mathrm{vHC}$ synapses in IL. Fear conditioning increased the ratio of AMPAR to NMDA receptor (NMDAR)-mediated currents by reducing NMDAR currents at the $\mathrm{VHC}$ synapses in IL. This postsynaptic plasticity was reversed more efficiently when extinction was conducted in the conditioning context than in a novel context. Furthermore, animals that showed poor extinction memory, a PTSD-like phenotype, failed to reverse the conditioning-induced plasticity, whereas the plasticity could be reversed by the ex vivo application of BDNF, which is known to simulate extinction when infused into IL (Peters et al., 2010; Rosas-Vidal et al., 2014).

\section{Materials and Methods}

\section{Animal subjects}

All animal procedures were approved by the Institutional Animal Care and Use Committee in compliance with 
NIH guidelines for the care and use of laboratory animals. Male Sprague Dawley rats were transported from the institutional colony to a satellite facility nearby where they were individually housed on a 12/12 h light/dark schedule with free access to food and water.

\section{Stereotaxic surgery}

Rats (between 90 and $120 \mathrm{~g}$ of weight) received bilateral injections of an AAV5 vector $(1.0 \mu \mathrm{l})$ expressing channelrhodopsin-2 (ChR2) and enhanced yellow fluorescent protein (EYFP) driven by the neuron-specific CaMKII promoter (AAV-CaMKIla-hChR2(H134R)-EYFP; University of North Carolina at Chapel Hill Vector Core Services) into the $\mathrm{vHC}$ via a $5-\mu \mathrm{l}$ Hamilton syringe using stereotactic coordinates (Sierra-Mercado et al., 2011). After waiting a period of 8-12 weeks for recovery and adequate ChR2 expression, rats received behavioral training. After completing behavioral training, animals were euthanized for acute brain slice preparation. In addition, brain sections containing the hippocampus were fixed and prepared for further confirmation of EYFP expression in the $\mathrm{vHC}$.

\section{Behavioral apparatus}

The fear conditioning context (context A) was a chamber of $25 \times 29 \times 28 \mathrm{~cm}$ with aluminum and Plexiglas walls (Coulbourn Inst.). The floor consisted of stainless-steel bars that could be electrified to deliver a mild shock and a single overhead light provided illumination. Context $B$, which was used to give extinction and recall test to the DIFF group, consisted of a hexagonal chamber with a flat floor, citric scent, and different illumination color. Contexts A and B had speakers mounted on the outside wall and were situated inside a sound-attenuating box (Med Associates) with a ventilating fan that produced an ambient noise level of 60 $\mathrm{dB}$. The conditioned stimulus (CS) was a $4-\mathrm{kHz}$ tone with duration of $30 \mathrm{~s}$ and an intensity of $80 \mathrm{~dB}$. The intertone interval for successive tone presentations in the conditioning, extinction, and test phases was an average of 2 min. The unconditioned stimulus (US) was a 0.50-mA scrambled foot shock, $0.5 \mathrm{~s}$ in duration, that co-terminated with the tone during the conditioning phase. Behavior was recorded with digital video cameras (Micro Video Products).

\section{Fear conditioning and extinction}

Animals were randomly assigned to one of the following experimental groups: pseudo-conditioned (PSEUDO), fear conditioned (COND), fear extinguished in context $A$ (SAME), or fear extinguished in context B (DIFF). On day 1, animals from the COND, SAME, and DIFF groups received auditory fear conditioning, which consisted of one habituation tone followed by five tone-shock pairings. Animals from the PSEUDO group received the same amount of tones and shocks but in a non-paired manner. On day 2, PSEUDO and COND animals remained in their home cages while SAME and DIFF animals received two sessions of 15 tone-alone presentations, with $1 \mathrm{~h}$ between sessions, in context $A$ and context $B$, respectively. On day 3 , rats from the PSEUDO, COND, and SAME groups received two tone-alone presentations in context $A$, whereas rats from the DIFF group received two tonealone presentations in context B. For the BDNF experi- ments, animals received fear conditioning on day 1 and recall test on day 2 .

\section{Whole-cell recordings}

Immediately after the test on day 3, rats were deeply anesthetized with pentobarbital $(65 \mathrm{mg} / \mathrm{kg})$ and perfused transcardially with ice-cold N-methyl-D-glucamine (NMDG) based artificial CSF (ACSF) and decapitated. Brains were quickly removed and placed in ice-cold NMDG ACSF. Then, $300-\mu \mathrm{m}$ coronal slices of the MPFC were cut with a Vibratome 1000 Plus (Vibratome). We used a modified NMDG-based ACSF to obtain healthy brain slices from adult animals. The composition of the NMDG-based ACSF (Ting et al., 2014) was the following: 93 mM NMDG, $2.5 \mathrm{mM} \mathrm{KCl}, 1.2 \mathrm{mM} \mathrm{NaH}_{2} \mathrm{PO}_{4}, 30 \mathrm{mM} \mathrm{NaHCO}, 20 \mathrm{mM}$ HEPES, $25 \mathrm{mM}$ glucose, $5 \mathrm{mM}$ sodium ascorbate, $2 \mathrm{mM}$ thiourea, $3 \mathrm{mM}$ sodium pyruvate, $10 \mathrm{mM} \mathrm{MgSO}_{4}$, and 0.5 $\mathrm{mM} \mathrm{CaCl} 2$. The mPFC slices were initially incubated at $33^{\circ} \mathrm{C}$ in NMDG ACSF for 12 min before being transferred to an additional $1-\mathrm{h}$ incubation in modified HEPES ACSF at room temperature $\left(21-23^{\circ} \mathrm{C}\right)$. The composition of the modified HEPES ACSF was the following: $92 \mathrm{mM} \mathrm{NaCl}$, $2.5 \mathrm{mM} \mathrm{KCl}, 1.2 \mathrm{mM} \mathrm{NaH}_{2} \mathrm{PO}_{4}, 30 \mathrm{mM} \mathrm{NaHCO}, 20 \mathrm{mM}$ HEPES, $25 \mathrm{mM}$ glucose, $5 \mathrm{mM}$ sodium ascorbate, $2 \mathrm{mM}$ thiourea, $3 \mathrm{mM}$ sodium pyruvate, $2 \mathrm{mM} \mathrm{MgSO}_{4}$, and 2 $\mathrm{mM} \mathrm{CaCl}_{2}$. Then, mPFC slices were transferred and submerged in the recording chamber and perfused at 2-3 $\mathrm{ml} / \mathrm{min}$ with room temperature ACSF with $1 \mu \mathrm{M}$ tetrodotoxin (TTX) and $100 \mu \mathrm{M}$ 4-amynopyridine (4-AP) to assess monosynaptic postsynaptic currents. In addition, $10 \mu \mathrm{M}$ picrotoxin was added to block $\mathrm{GABA}_{\mathrm{A}}$ postsynaptic currents. The composition of the recording ACSF was the following: $126 \mathrm{mM} \mathrm{NaCl}, 3 \mathrm{mM} \mathrm{KCl}, 1.25 \mathrm{mM} \mathrm{NaH}_{2} \mathrm{PO}_{4}, 1$ $\mathrm{mM} \mathrm{MgSO}_{4}, 26 \mathrm{mM} \mathrm{NaHCO}_{3}, 20 \mathrm{mM}$ glucose, and $2 \mathrm{mM}$ $\mathrm{CaCl}_{2}$ and bubbled with $95 \% \mathrm{O}_{2}$ and $5 \% \mathrm{CO}_{2}$. The neurons were visualized with infrared video microscopy using a $40 \times$ water-immersion objective on an upright E600FN microscope (Nikon Instruments). Whole-cell recordings were done with glass pipettes with a resistance of 2.5-4 $\mathrm{M} \Omega$ and filled with cesium gluconate internal solution containing the following: $12 \mathrm{mM}$ TEA-Cl, $140 \mathrm{mM} \mathrm{CsOH}$, 10 mM HEPES, 140 mM gluconic acid, 10 mM biocytin, 2 $\mathrm{mM}$ adenosine triphosphate, $3 \mathrm{mM}$ guanosine triphosphate, and $0.4 \mathrm{mM}$ cesium-ethylene glycol-bis(2aminoethylether)-N,N,N',N'-tetra acetic acid (Cs-EGTA, 0.4); $\mathrm{pH}$ was adjusted to 7.3 with $\mathrm{CsOH}$ (300 mOsm). After establishing a whole-cell voltage-clamp recording, the membrane resistance, membrane capacitance, and access resistance were measured. Recordings were filtered at $4 \mathrm{kHz}$, digitized at $10 \mathrm{kHz}$, and saved to a computer using pCLAMP9 (Molecular Devices).

\section{AMPA and NMDAR currents}

AMPA and NMDAR-mediated EPSCs in mPFC neurons were measured in response to the optical stimulation of ChR2-expressing vHC axons innervating Layer II/III and V with a 470-nm light-emitting diode (LED; M470L2, Thorlabs) through the $40 \times$ objective centered at the soma of the patched neuron with light intensity of $1.5 \mathrm{~mW}$. A total of 106 neurons were recorded from IL $(89 \%$ in Layer V; $11 \%$ in Layer II/III). Picrotoxin was added to the bath to 
block $\mathrm{GABA}_{\mathrm{A}}$-mediated currents. TTX and 4-AP were added to ensure monosynaptic measurements. To evoke synaptic responses in the mPFC by photostimulation of $\mathrm{vHC}$ fibers, we illuminated slices every $10 \mathrm{~s}$ with light pulses of 5- to 20-ms duration. AMPAR-mediated EPSCs were measured as the peak of the EPSCs recorded at -70 $\mathrm{mV}$. NMDAR-mediated EPSCs were measured as the amplitude of the EPSC at $+40 \mathrm{mV}, 70 \mathrm{~ms}$ after the light stimulus. Initial experiments with NMDAR blockers showed that EPSCs contained minimal contamination with AMPAR EPSCs at this time point. Measurements of AMPAR and NMDAR EPSCs were also taken at $0 \mathrm{mV}$ to calculate ion conductance from the current (I) versus voltage $(\mathrm{V})$ plot and peak values were taken similarly to at -70 and $+40 \mathrm{mV}$. AMPAR conductance values were calculated by taking the slope of the I/N plot of AMPA EPSCs measured at holding potentials of -70 and $0 \mathrm{mV}$, whereas NMDAR conductance values were calculated from the I/V plot values of NMDAR EPSCs measured at holding potentials of 0 and $+40 \mathrm{mV}$.

\section{BDNF experiments}

We prepared $\mathrm{mPFC}$ slices from rats that received fear conditioned on day 1 and a fear recall test on day 2 . The slices underwent the same recovery process as described above in the whole-cell recordings section. After the slice recovery period, mPFC slice hemispheres were separated and one hemisphere was randomly selected for an additional 1-h incubation in room temperature HEPES-ACSF containing 2 nM BDNF (Invitrogen). The other hemisphere was incubated an additional hour in control HEPESACSF. After the 1-h incubation with BDNF or control HEPES-ACSF, slices were transferred to the recording chamber and AMPAR- and NMDAR-mediated EPSCs evoked by optical stimulation of $\mathrm{vHC}$ axons were recorded in IL pyramidal neurons.

\section{Histology}

In all experiments, $10 \mathrm{mM}$ biocytin was included in the recording solution to label the neurons for post hoc morphologic identification of IL pyramidal neurons. After completing electrophysiological recordings, the slices were fixed overnight in 4\% paraformaldehyde. Recorded IL neurons were stained with a standard avidin-biotin peroxidase procedure (Vectastain ABC kit; Vector Laboratories) and visualized with bright-field microscopy. Neurons that were not located in the IL or that were not pyramidal-shaped with obvious apical dendrites were excluded from the analysis.

\section{Statistical analysis}

Freezing behavior was used as an indicator of fear and was assessed uniformly using computer-based analysis program (FreezeScan, Clever Systems). All behavioral data were compared with repeated measures ANOVA followed by Tukey HSD post hoc test (IBM SPSS Statistics, IBM Corp.). The electrophysiological data were analyzed using Clampfit (Molecular Devices) and were compared with nonparametric Kruskal-Wallis test or Mann-Whitney test due to skewness of the data in its distribution (IBM SPSS Statistics, IBM Corp.). Significant main effect with Kruskal-Wallis test was followed by Dunn's post hoc test. Effect sizes between means were assessed using Cohen's $d$ (Cohen, 1988; Lenhard and Lenhard, 2016). Values are reported as the mean \pm SEM. All animals in which we were able to obtain stable optically-evoked EPSCs were included in the behavioral analysis. Since we wanted to correlate synaptic changes with behavioral changes, we analyzed rats from the extinction groups that showed $<50 \%$ freezing during the extinction recall tones on day 3 (successful extinction) separate from those rats that failed to recall extinction (unsuccessful) and froze $>50 \%$ during the recall tones on day 3 . Seven of the 13 rats that were extinguished in the fear conditioning context showed successful extinction recall in the conditioning context. All seven rats that received extinction in the novel context showed successful extinction recall in the novel context.

\section{Results}

First, AAV vectors expressing ChR2 and EYFP were injected into the $\mathrm{vHC}$ of rats. Two to three months later, robust expression of the viral proteins could be seen in the $\mathrm{vHC}$ and in the axons in the mPFC (Fig. 1A,B). Optical stimulation of ChR2-expressing $\mathrm{vHC}$ axons evoked EPSCs in IL pyramidal neurons. Whole-cell recordings at -70 $\mathrm{mV}$ allowed the measurement of AMPA receptormediated EPSCs from the peak current (Fig. 1C). Optical stimulation at $+40 \mathrm{mV}$ evoked both AMPA and NMDAR currents. Initial experiments with NMDAR blockers showed that $70 \mathrm{~ms}$ after stimulation the NMDAR EPSCs contained minimal contamination with AMPAR EPSCs (Fig. 1C). Next, we designed four experimental groups to test whether fear conditioning or extinction induces changes in $\mathrm{vHC}$ synapses in IL, and whether extinction in the same context (SAME) will induce different synaptic changes in $\mathrm{VHC}$ synapses than extinction in a novel context (DIFF; Fig. 1D). On day 1, the group that received conditioning but no extinction (COND, $n=10$ ) and the SAME $(n=7)$ and DIFF $(n=7)$ groups acquired similar levels of fear after receiving auditory fear conditioning in context A (Fig. 2A). The PSEUDO $(n=7)$ group received unpaired tone and shock presentations in context $A$ to avoid association of the tone with the shock. Then, on days 2 and 3 , the SAME group received fear extinction and extinction retrieval test in context $A$ whereas the DIFF group received extinction and retrieval test in context $B$. On day 3, we tested all animals for cued fear and euthanized animals immediately after the test. A cohort of rats in the SAME group were removed from the analysis due to poor extinction memory recall on day 3 and were analyzed separately in Figure 4. As expected (Fig. 2A), a repeated measures ANOVA showed a significant main effect $\left(F_{(2,12)}\right.$ $=40.63, p<0.001$ ) and post hoc analysis confirmed that rats from the COND group had significantly higher levels of freezing to the tone on day 3 compared to rats from the SAME, DIFF, and PSEUDO groups $(p<0.001)$. The difference in fear expression among groups indicates that all animals were successful in learning their respective behaviors. It is important to point out that the DIFF group received extinction and extinction recall in a novel context. Therefore, the DIFF group did not receive extinction of the conditioning context. Given the abundant evidence 
A Injection Site

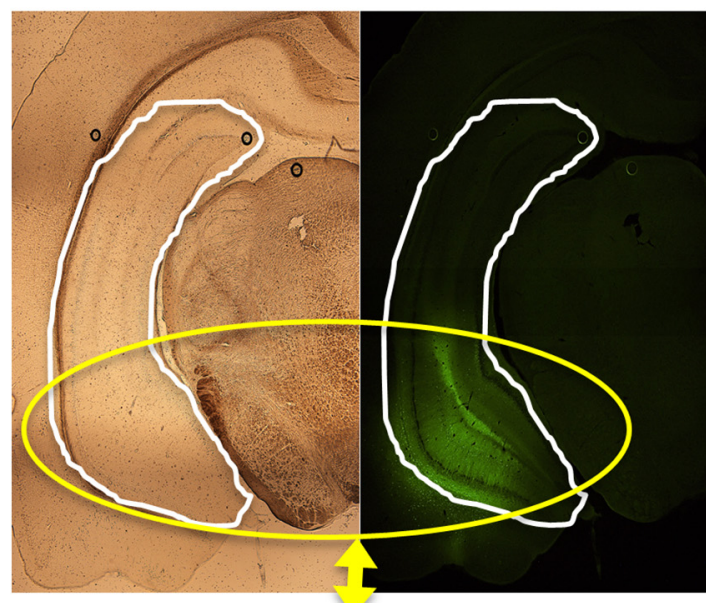

Ventral hippocampus

\section{B Projection/Recording Site}

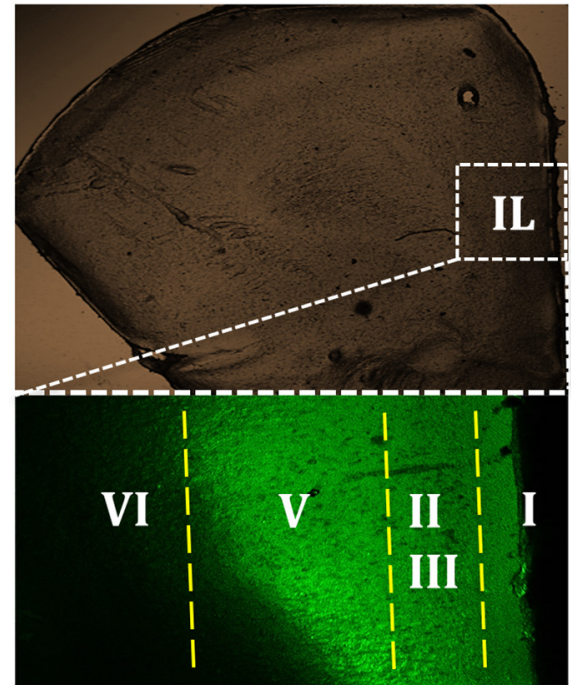

C

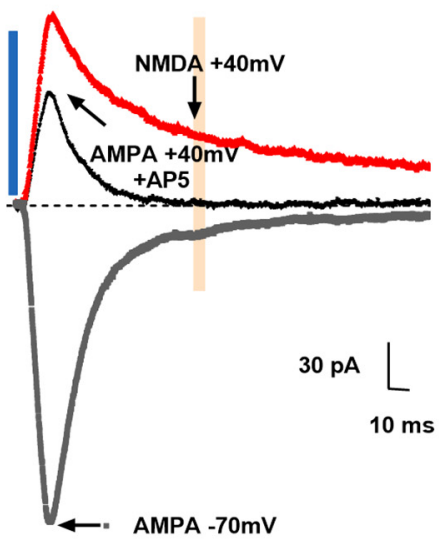

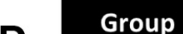

\begin{tabular}{|c|c|c|c|}
\hline Group & Day 1 & Day 2 & Day 3 \\
\hline PSEUDO & $\begin{array}{c}6 \text { tones } \\
5 \text { shocks } \\
\text { no } \\
\text { pairings } \\
\text {-Context A }\end{array}$ & \multirow[t]{2}{*}{ Home cage } & \multirow[t]{2}{*}{$\begin{array}{c}2 \text { tone alone } \\
\text { presentations } \\
\text { Context A }\end{array}$} \\
\hline COND & \multirow[b]{2}{*}{$\begin{array}{l}5 \text { tone- } \\
\text { shock } \\
\text { pairings }\end{array}$} & & \\
\hline SAME & & $\begin{array}{c}2 \times 15 \text { tone alone } \\
\text { presentations } \\
\text { Context } A\end{array}$ & $\begin{array}{c}2 \text { tone alone } \\
\text { presentations } \\
\text { Context } \mathrm{A}\end{array}$ \\
\hline DIFF & Context A & $\begin{array}{c}2 \times 15 \text { tone alone } \\
\text { presentations } \\
\text { Context B }\end{array}$ & $\begin{array}{c}2 \text { tone alone } \\
\text { presentations } \\
\text { Context B }\end{array}$ \\
\hline
\end{tabular}

E

\section{Behavioral experiments}

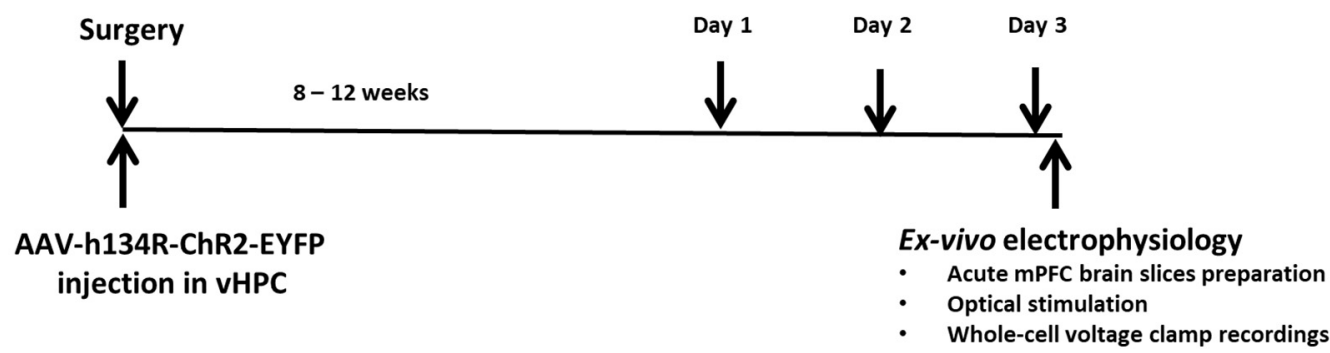

Figure 1. Experimental design to measure vHC EPSCs in IL neurons after fear conditioning and extinction. $\boldsymbol{A}, \boldsymbol{B}, \mathrm{ChR} 2$ expression in the $\mathrm{vHC}$ and $\mathrm{mPFC}$. $\boldsymbol{C}$, AMPAR-mediated EPSCs were measured at the peak of the $-70-\mathrm{mV}$ current. NMDAR-mediated EPSCs were measured $70 \mathrm{~ms}$ after stimulation to minimize the possibility of contamination with AMPAR EPSC. $\boldsymbol{D}$, Behavioral groups. $\boldsymbol{E}$, Scheme of the general timeline for the experiments.

of fear renewal (Bouton et al., 2006), the DIFF group would likely show higher freezing in the conditioning context.

Fear conditioning and extinction induce plasticity in vHC-IL synapses

After recall on day 3 , we prepared acute brain slices and used whole-cell voltage-clamp recordings to assess
AMPA to NMDA ratios in MPFC pyramidal neurons after selective optical stimulation of $\mathrm{vHC}$ axons (Fig. $2 B, C$ ). The following number of cells was evaluated in each group: PSEUDO ( $n=22$ cells from seven rats), COND ( $n=25$ cells from 10 rats), SAME ( $n=23$ cells from seven rats), and DIFF ( $n=20$ cells from seven rats). Kruskal-Wallis test showed a significant main effect in AMPA to NMDA 

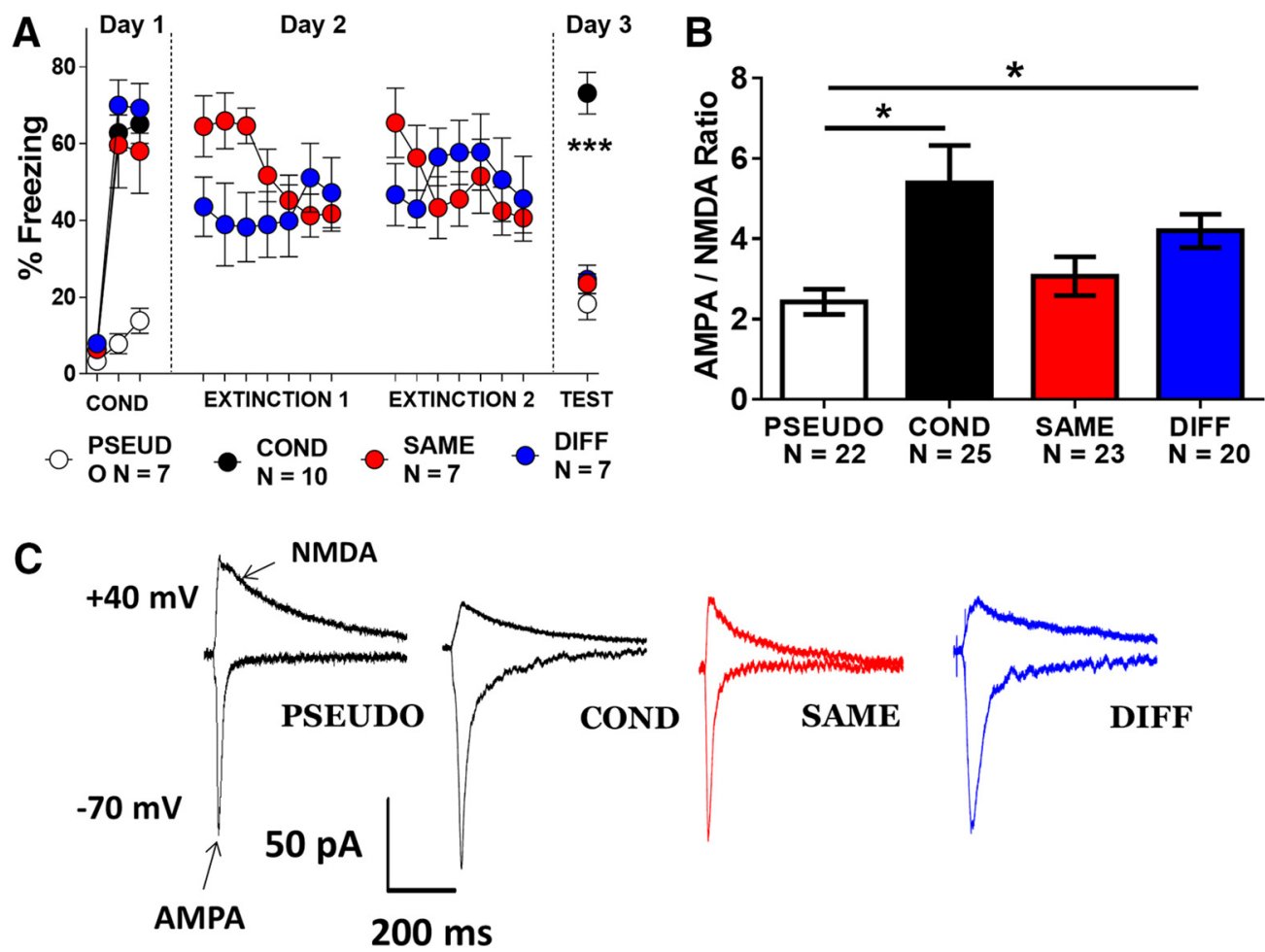

Figure 2. Fear conditioning and extinction induce postsynaptic plasticity in vHC synapses onto IL pyramidal neurons. $\boldsymbol{A}$, Behavioral responses of all groups. $\boldsymbol{B}$, Mean AMPA/NMDA ratios measured in IL pyramidal neurons from each group. $\boldsymbol{C}$, Example traces of vHC EPSCs recorded in IL pyramidal neurons of each group $(\boldsymbol{A}, * * * p<0.001$, repeated measures ANOVA, COND vs PSEUDO, SAME, and DIFF; $\boldsymbol{B}, * p<0.05$, Dunn's post hoc after significant Kruskal-Wallis test).

ratios of $\mathrm{vHC}$ inputs in $\mathrm{IL}\left(H_{(3,90)}=14.70, p=0.002\right)$. Dunn's post hoc analysis revealed a significant increase in AMPA to NMDA ratios in the COND $(p=0.012)$ and DIFF $(p=0.018)$ groups compared to the PSEUDO group but not the SAME group (Fig. $2 B$; PSEUDO vs SAME, $p=$ 1.00 ; COND vs SAME, $p=0.12$; COND vs DIFF, $p=1.00$; SAME vs DIFF, $p=0.15)$. Moreover, large effect sizes in AMPA to NMDA ratios were found between the COND $(d$ $=0.8)$ and the DIFF $(d=1.1)$ groups compared to the PSEUDO group. SAME versus COND $(d=0.6)$ and SAME versus DIFF $(d=0.5)$ showed intermediate effect sizes. Small effect sizes were found between PSEUDO versus SAME $(d=0.3)$ and COND versus DIFF $(d=0.3)$. Therefore, a significant reversal of the AMPA to NMDA ratio increase found in the COND group was observed only in the SAME group. These findings suggest that fear conditioning induces postsynaptic plasticity of $\mathrm{vHC}$ inputs in IL that is more effectively reversed when extinction occurs in the same context as conditioning.

\section{Fear conditioning and extinction alter NMDAR currents in vHC-IL synapses}

Since changes in either AMPAR or NMDAR-mediated EPSCs could produce a change in the AMPA to NMDA ratios, we evaluated the AMPAR and NMDAR components separately to determine which one was responsible for the changes observed in IL neurons (Fig. 3). First we examined the AMPA component and found no significant differences in AMPAR EPSCs $\left(H_{(3,90)}=5.48, p=0.14\right)$ or conductance $\left(H_{(3,90)}=1.95, p=0.58\right.$; Fig. $\left.3 A, B\right)$. In contrast, we found that fear conditioning significantly reduced NMDAR EPSCs and extinction reversed the reduction (Fig. $3 C$ ). Kruskal-Wallis test showed a significant main effect in NMDAR EPSCs $\left(H_{(3,90)}=15.98, p=0.001\right)$ and post hoc analysis revealed that NMDAR EPSCs were smaller in the COND group compared to PSEUDO $(p=$ $0.002)$ and SAME $(p=0.023)$ groups (COND vs DIFF, $p=$ 1.00; PSEUDO vs SAME, $p=1.00$; PSEUDO vs DIFF, $p=$ 0.12 ; SAME vs DIFF, $p=0.65$ ). Cohen's $d$ found intermediate effect sizes in NMDAR EPSCs in PSEUDO versus COND $(d=0.7)$, PSEUDO versus DIFF $(d=0.5)$, COND versus SAME $(d=0.6)$ and SAME versus DIFF $(d=0.5)$. Small effect sizes were found in COND versus DIFF $(d=$ $0.3)$, and no effect in PSEUDO versus SAME $(d=0.01)$. In addition, we found that fear conditioning decreased NMDAR conductance and extinction reversed the decrease in NMDAR conductance (Fig. 3D). Kruskal-Wallis test showed a significant main effect in NMDAR conductance $\left(H_{(3,90)}=14.26, p=0.003\right)$ and post hoc analysis revealed that NMDAR conductance was smaller in the COND group compared to PSEUDO $(p=0.003)$ and SAME $(p=0.023)$ groups (COND vs DIFF, $p=0.53$; PSEUDO vs SAME, $p=1.00$; PSEUDO vs DIFF, $p=0.60$; SAME vs DIFF, $p=1.00$ ). Cohen's $d$ found intermediate effect sizes in NMDAR conductance in COND versus PSEUDO $(d=0.7)$ and COND versus SAME $(d=0.7)$. Small effect sizes were found in PSEUDO versus DIFF ( $d$ $=0.4)$, COND versus DIFF $(d=0.4)$ and SAME versus DIFF $(d=0.4)$. These results suggest that the changes observed in AMPA to NMDA ratios were caused by alter- 
A
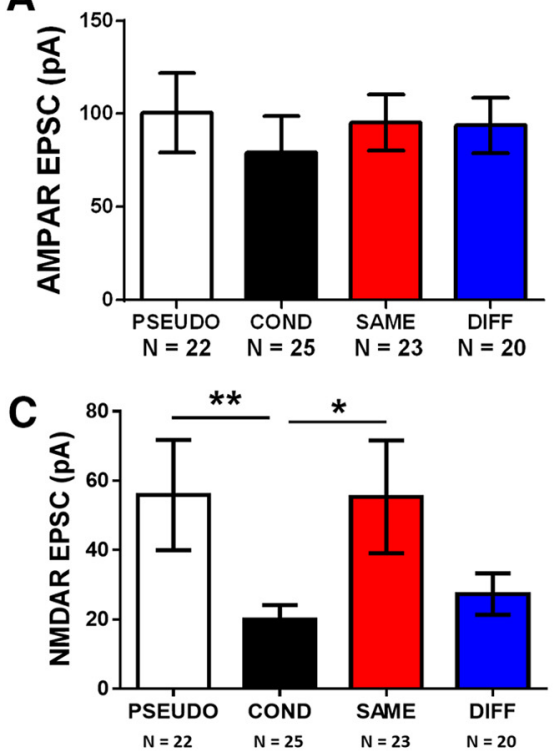
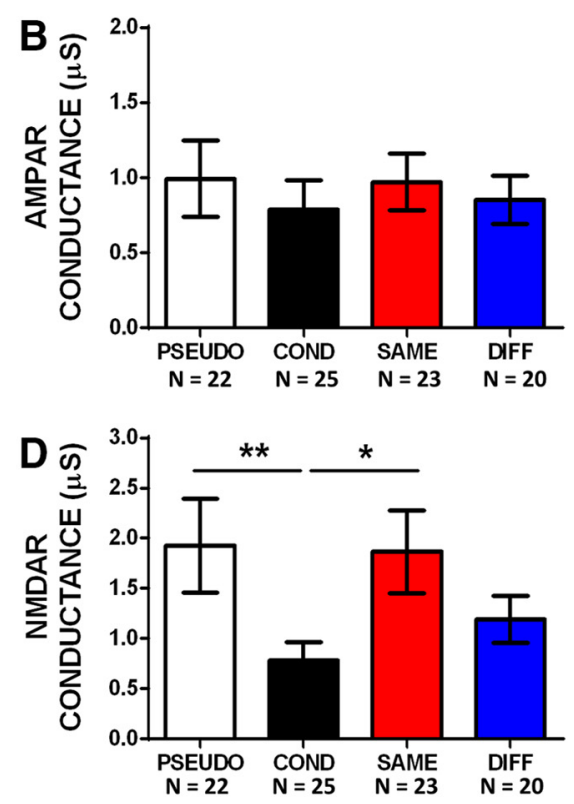

Figure 3. Fear conditioning and extinction induced bidirectional changes in NMDAR currents at vHC synapses onto IL pyramidal neurons. $\boldsymbol{A}, \boldsymbol{B}$, Average AMPAR-mediated EPSCs and conductance in each group. $\boldsymbol{C}, \boldsymbol{D}$, Average NMDAR-mediated EPSCs and conductance in each group $(* * p<0.01, * p<0.05$; Dunn's post hoc after significant Kruskal-Wallis test).

ations in NMDAR mediated currents induced by fear conditioning and extinction. Acquisition of fear reduced the NMDAR-mediated currents at vHC synapses onto IL neurons and extinction in the same context reversed this depression. Extinction in a novel context failed to reverse the depressed NMDAR currents.

\section{Failure to recall extinction correlates with failure to reverse conditioning-induced depression of NMDAR currents in vHC-IL synapses}

As found previously by others (Burgos-Robles et al., 2007; Peters et al., 2010; Gruene et al., 2015), we found a cohort of animals that received extinction in the fear conditioning context (SAME, from Fig. 2A) that showed poor extinction memory retrieval (UNSUCCESSFULL; Fig. $4 A)$. Thus, although these animals received the same behavioral treatment as the SAME group in Figure $2 A$, they showed poor extinction memory with $>50 \%$ freezing during the extinction recall test (UNSUCCESSFUL group; Fig. 4A). In fact, the UNSUCCESSFULL group behaved as though it never received extinction and froze as much as the COND group at recall $(t=0.07634, \mathrm{df}=14, p=$ 0.9402). In comparison, the SAME group from Figure $2 A$ showed $<50 \%$ freezing during recall (SUCCESSFUL group; Fig. 4A). If the reversal of NMDAR currents by extinction is important for extinction memory, then animals that failed to remember extinction should show smaller NMDAR currents. Consistent with this, AMPA to NMDA ratios of $\mathrm{vHC}$ inputs in IL of the UNSUCCESSFUL ( $n=16$ cells from six rats) group were greater than those from rats with successful extinction retrieval (SUCCESSFUL, $n=23$ cells from seven rats; Mann-Whitney $U$ test, $U=108, p=0.030$ ). In addition, a large effect size was found in AMPA to NMDA ratios between the groups $(d=$ 0.8). As observed in COND animals (Fig. 3D), the larger AMPA to NMDA ratios in the UNSUCCESSFUL group was caused by smaller NMDAR EPSCs (Fig. 4D,E). MannWhitney $U$ test showed that the UNSUCCESSFUL group had smaller NMDAR EPSCs $(U=266, p=0.019)$ with an intermediate effect size $(d=0.6)$. Also, NMDAR conductance in UNSUCCESSFUL animals showed a tendency toward a reduction $(U=248, p=0.069)$ with an intermediate effect size $(d=0.6)$. Once again, no differences were found in the AMPAR current $(U=216, p=0.37)$ or conductance $(U=192, p=0.83$; Fig. $4 F, G)$. These findings suggest that the observed changes in NMDAR currents at vHC synapses in IL are important for successful extinction memory retrieval.

During auditory fear conditioning, the rats learn to associate the tone and context with the aversive foot shock. Therefore, differences in contextual fear among the behavioral groups may also contribute to the synaptic differences. To address this issue, we calculated the percentage of time the rats froze during the minute before the first tone on day 3 which is a measure of fear to the context A for the PSEUDO $(n=7)$, COND $(n=10)$, SUCCESSFUL $(n=7)$, and UNSUCCESSFUL $(n=6)$ groups. The DIFF group that received extinction in a novel context $B$ were not included since their recall was given in context $\mathrm{B}$ and not in the fear conditioning context A. Although the contextual fear varied among the groups (Fig. $5 A)$, there were no significant differences among them $\left(F_{(3,25)}\right.$ $=2.394, p=0.092)$. However, the small sample size likely affected this statistical analysis; therefore, we also calculated effect size. When we evaluated the effect size, we found a large overall effect in contextual freezing among groups $(d=1.0)$. A large effect in contextual freezing was observed in the COND group compared to the PSEUDO ( $d$ $=0.9)$ and SAME $(d=0.8)$ groups. An intermediate effect in contextual freezing between the PSEUDO $(d=0.6)$ and SAME $(d=0.5)$ groups compared to the UNSUCCESFUL 
A1

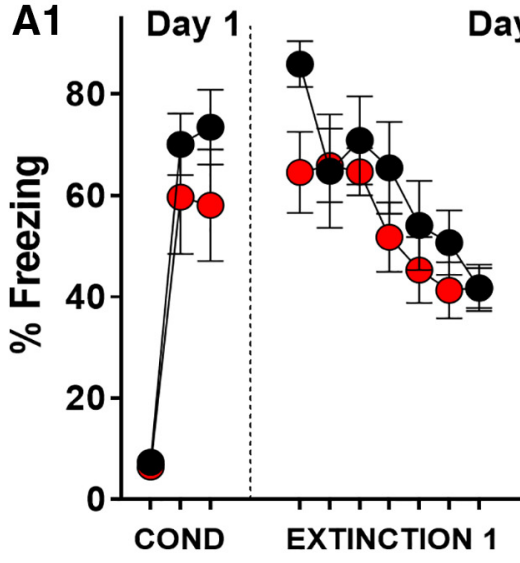

SAME SUCCESSFUL $\mathrm{N}=7$

B SAME SUCCESSFUL

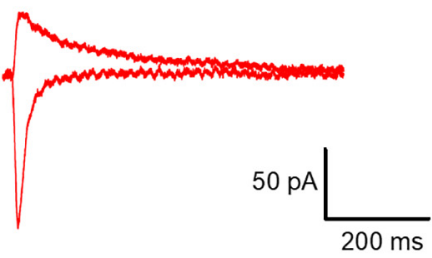

A2

Day 3

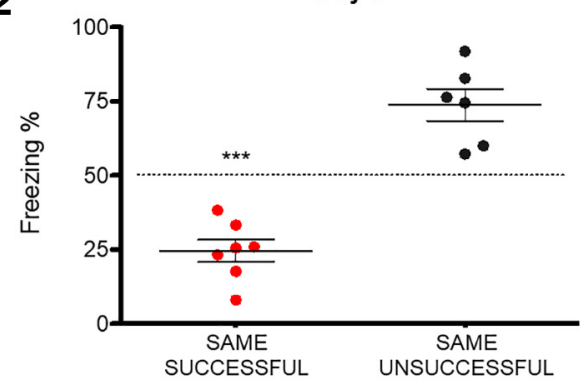

A3

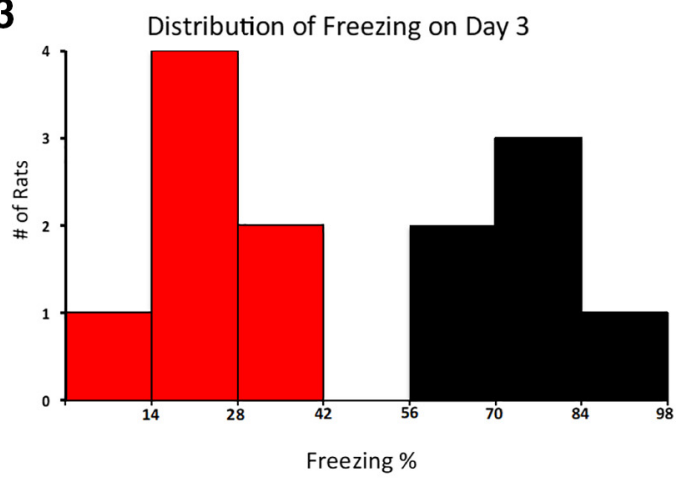

C

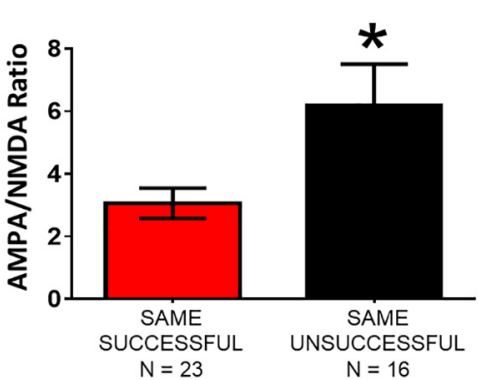

F

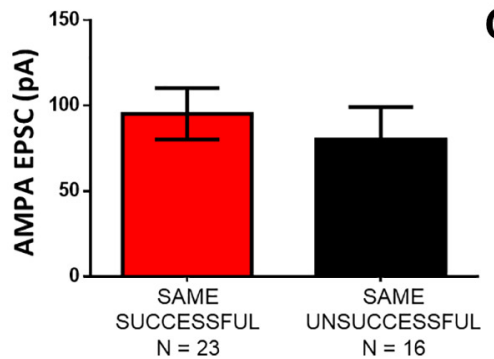

SAME UNSUCCESSFUL

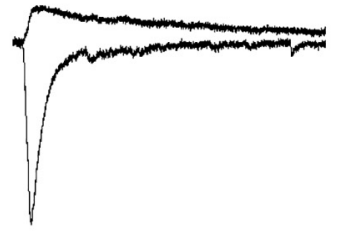

SAME UNSUCCESSFUL

$\mathrm{N}=6$

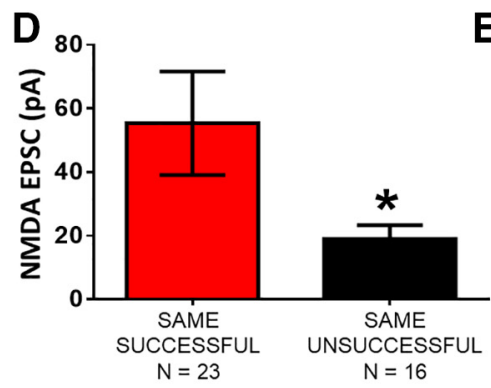

G

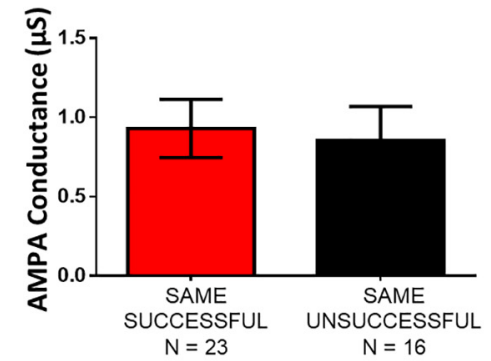

Figure 4. IL neurons from animals that failed to recall extinction showed greater AMPA/NMDA ratios and reduced NMDAR-mediated currents. $\boldsymbol{A}$, Behavioral data from same context extinction animals that showed successful or failed extinction recall. $\boldsymbol{B}$, Example vHC EPSCs recorded in IL pyramidal neurons. $\boldsymbol{C}$, Average AMPA/NMDA ratio in each group. $\boldsymbol{D}$, $\boldsymbol{E}$, Average NMDAR-mediated EPSC and conductance in each group. $\boldsymbol{F}, \mathbf{G}$, Average AMPAR-mediated EPSC and conductance in each group $(* p<0.05$; Mann-Whitney's test).

group. A small effect between the COND group compared to the UNSUCCESSFUL group $(d=0.2)$ and no effect between the PSEUDO and SAME groups $(d=0.1)$. Therefore, as expected the COND and UNSUCCESSFUL groups showed more contextual fear than the PSEUDO and SAME groups. Furthermore, the contextual fear shown on day 3 strongly correlated with the cued fear to the tones (Fig. 5B).
BDNF mimicked fear extinction by reversing fear conditioning-induced increase in AMPA/NMDA ratios and decrease in NMDAR currents in VHC-IL synapses

Previous studies have nicely demonstrated that in vivo infusion of BDNF into IL before extinction is sufficient to decrease fear expression and induce extinction (Peters 
A

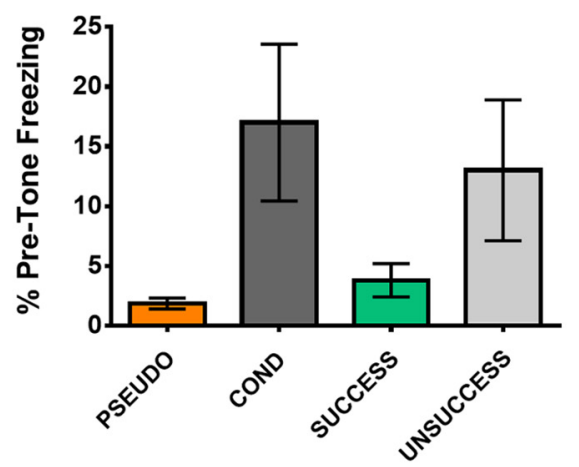

B

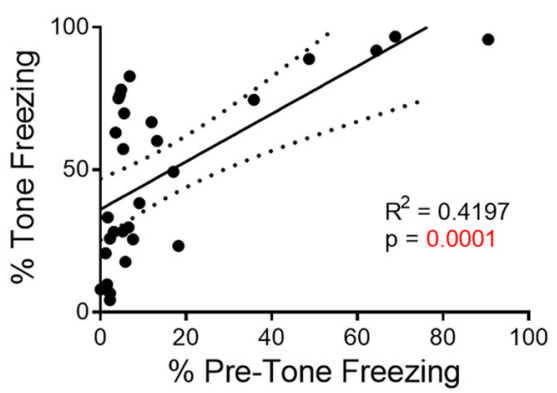

Figure 5. Contextual fear to context A varies with fear to tones. A, Average freezing during the minute before giving the first test tone on day 3 in the different groups. $\boldsymbol{B}$, Correlation between freezing before the tones and during the tones on day 3 . Rats from the PSEUDO, COND, SUCCESSFUL, and UNSUCCESSFUL groups are plotted.

et al., 2010; Rosas-Vidal et al., 2014). Therefore, if the changes we observed in the NMDAR currents are important for extinction, then BDNF should produce similar changes in the NMDAR currents at the vHC to IL synapses as fear extinction. As shown in Figure 6, we found that BDNF-treated IL neurons ( $n=11$ cells from four rats) from fear conditioned animals showed lower AMPA NMDA ratios and larger NMDAR EPSCs than non-treated IL neurons ( $n=9$ cells from four rats) from the same animals. Mann-Whitney $U$ test showed a significant mean difference in AMPA/NMDA ratios $(p=0.022)$ and NMDAR EPSCs $(p=0.033)$. Cohen's $d$ found large effects in AMPA/NMDA ratios $(d=1.2)$ and NMDAR EPSCs $(d=$ 0.8 ) between groups. Thus, ex vivo incubation with BDNF decreased the AMPA/NMDA ratio and increased the NMDAR currents in vHC-IL synapses mimicking the synaptic effects produced by fear extinction. These findings further substantiate that the observed changes in NMDAR currents at $\mathrm{vHC}-\mathrm{IL}$ synapses contribute to the behavioral changes.

\section{Discussion}

By using optogenetic stimulation, we found that auditory fear conditioning induces postsynaptic plasticity at $\mathrm{vHC}$ synapses onto IL pyramidal neurons that involves an increase in the ratio of AMPA to NMDAR currents. Further examination revealed that fear acquisition reduced the NMDAR currents without altering the AMPA currents. Previous studies using electrical stimulation of all axons within the vicinity of the electrode did not observe synaptic plasticity after fear conditioning in IL (Pattwell et al., 2012; Sepulveda-Orengo et al., 2013). This suggests that the depression of NMDAR currents at $\mathrm{VHC}$-IL synapses does not occur at the majority of synapses onto IL neurons and could be a unique feature of $\mathrm{vHC}$ synapses. Although we used established coordinates for infusion of the viral constructs into the rat vHC (Sierra-Mercado et al., 2011) and we confirmed expression of viral proteins in the $\mathrm{vHC}$ (Fig. 1A), it is not possible to completely rule out the possibility that some fibers from viral expression in areas near the $\mathrm{vHC}$ also contribute to the inputs recorded in IL.

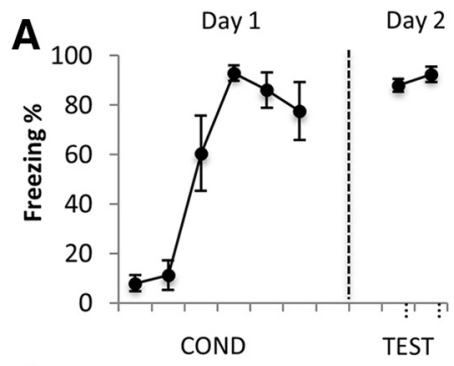

B
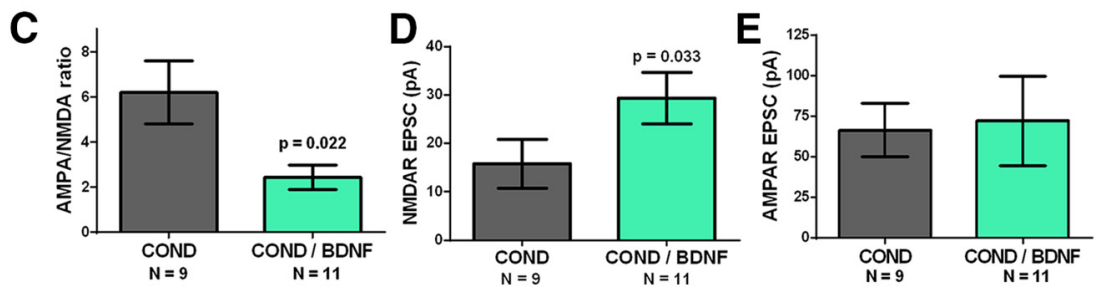

Figure 6. In vitro BDNF treatment mimicked fear extinction reversal of conditioning-induced changes in AMPA/NMDA ratio and NMDAR currents. A, Behavioral data. B, Example trace for each group. $\boldsymbol{C}$, Average AMPA/NMDA ratio for each group. $\boldsymbol{D}$, Average NMDAR EPSC for each group. $\boldsymbol{E}$, Average AMPAR EPSCs ( $p<0.05$, Mann-Whitney's test). 
However, based on the relatively robust projections from the vHC to IL (Hoover and Vertes, 2007), the majority of inputs activated in IL likely come from the vHC.

Exposure to extinction reversed the fear conditioninginduced plasticity leading to an increase in NMDAR currents and a corresponding decrease in AMPA/NMDA ratio. In support of our findings, a recent study in mice also found increased NMDA currents at VHC-IL synapses after fear extinction (Wang et al., 2018). In our experiments, extinction induced a more complete reversal of conditioning-induced plasticity when the extinction was conducted in the fear conditioning context. The fact that the group that received cued fear extinction in the novel context showed intermediary changes in the NMDAR EPSCs compared to the group that received extinction in the same context as fear conditioning suggests that extinction of fear to the cue and extinction of fear to the context both contribute to the synaptic modifications of the vHC-IL synapses. Exposure to extinction in the novel context likely left remnants of the contextual fear which produced NMDAR EPSCs in between those of the COND group and the group that received extinction in the fear conditioning context. This suggests that contextual and cued fear modulate vHC-IL synapses independently. Whether the same or independent populations of ventral hippocampal neurons are modulated remains to be determined.

The observed changes in AMPA/NMDA ratio, NMDAR EPSC, and NMDAR conductance at the VHC-IL synapse varied with the levels of fear expression suggesting that these changes contribute to the behavioral outcome. The fact that animals that failed to recall extinction memory did not show a reversal of the conditioning-induced changes suggests that the changes in NMDAR requires the behavioral change rather than simple exposure to extinction training. Moreover, the extinction-induced changes were mimicked by incubating slices with BDNF. Therefore, plasticity of NMDAR currents at $\mathrm{vHC}$ to $\mathrm{IL}$ synapses appears to modulate cued fear expression and represents a novel mechanism for modulating conditioned fear.

Our findings suggest that learning-induced changes in NMDAR currents occur in response to the acquisition and extinction of conditioned fear. Consistent with our findings, physiologically relevant stimuli in brain slices can induce selective changes in NMDAR currents without altering AMPA receptor currents (Kwon and Castillo, 2008; Rebola et al., 2008). Furthermore, NMDAR show bidirectional plasticity in response to synaptic stimulation in slices (Rebola et al., 2010; Hunt and Castillo, 2012). Similar to our findings, fear learning decreased NMDAR currents in the amygdala by reduced phosphorylation of the GluN1 subunit of NMDAR (Zinebi et al., 2003). Although acute stress can depress NMDAR currents (Kuzmiski et al., 2010), it is unlikely that the changes in NMDAR currents we observed were mediated by stress, since our results were compared to the pseudoconditioned group that should have similar stress exposure.
Previous studies demonstrated that infusion of BDNF into IL is sufficient to simulate extinction and reduce fear to a CS (Peters et al., 2010; Rosas-Vidal et al., 2014). This effect of BDNF was prevented by systemic blockade of NMDARs (Peters et al., 2010) suggesting that BDNF may increase NMDA currents to reduce conditioned fear. Consistent with this possibility, we found that ex vivo incubation of prefrontal slices with BDNF induced similar synaptic changes in the $\mathrm{VHC}$ inputs in IL as extinction. Another recent study in mice also showed that incubation with BDNF increases the NMDA currents at vHC synapses in IL (Wang et al., 2018). Therefore, one synaptic mechanism by which BDNF simulates extinction may be by increasing NMDAR currents at $\mathrm{VHC}$ inputs in IL pyramidal neurons. However, an infusion of BDNF in vivo may also induce additional cellular and circuit effects that contribute to the reduction in conditioned fear.

Inputs from $\mathrm{VHC}$ to the MPFC provide spatial representations that guide aversive behaviors (Padilla-Coreano et al., 2016). Our findings suggest that the behavioral outcome of $\mathrm{VHC}$ stimulation of IL is altered by fear conditioning and extinction. Consistent with IL's proposed role of inhibiting freezing in response to aversive cues (Milad and Quirk, 2002; Sierra-Mercado et al., 2011; Moscarello and LeDoux, 2013), weakened vHC activation of IL pyramidal neurons after fear conditioning would increase freezing behavior. This reduced synaptic activation of IL excitatory neurons combined with the strong feedforward inhibition produced by vHC inputs in IL (Marek et al., 2018) and depressed intrinsic excitability of IL pyramidal neurons after fear conditioning (Santini et al., 2008; SolerCedeño et al., 2016) would reduce IL activation of downstream targets such as the amygdala (Quirk et al., 2003; Cho et al., 2013) and enhance acquisition of conditioned fear. In support of this model, stimulation of IL excitatory neurons impairs the acquisition of conditioned fear (Yizhar et al., 2011) as does increased glucocorticoid signaling in IL (Criado-Marrero et al., 2017).

After fear extinction, the increase in $\mathrm{vHC}$ activation of NMDAR currents on IL neurons would increase the activation of IL pyramidal neurons (Orsini et al., 2011; Kim and Cho, 2017). The slower kinetics of NMDARs allow them to drive neuronal burst firing (Polsky et al., 2009; Grienberger et al., 2014). Therefore, the relative increase in NMDAR currents after extinction in the conditioning context likely contributes to the increase in NMDAR-dependent burst firing seen in IL neurons shortly after acquisition of fear extinction which correlated with good extinction memory (Burgos-Robles et al., 2007). The resulting increased activation of the amygdala by IL projections would produce a context-dependent modulation of conditioned fear (Orsini et al., 2011; Maren et al., 2013).

In conclusion, we have found that fear conditioning and extinction induce bidirectional changes in NMDAR currents at $\mathrm{VHC}$ synapses in IL. Acquisition of conditioned fear reduced NMDAR currents, while extinction enhanced the NMDAR currents. Failure to reverse the conditioninginduced depression of NMDAR currents led to poor extinction memory and a PTSD-like phenotype. Medications designed to activate BDNF receptors may be useful for 
enhancing NMDAR currents at $\mathrm{VHC}$ synapses in $\mathrm{IL}$ and treating PTSD.

\section{References}

Bouton ME (2004) Context and behavioral processes in extinction. Learn Mem 11:485-494.

Bouton ME, Bolles RC (1979) Contextual control of the extinction of conditioned fear. Learn Motiv 10:445-466.

Bouton ME, Westbrook RF, Corcoran KA, Maren S (2006) Contextual and temporal modulation of extinction: behavioral and biological mechanisms. Biol Psychiatry 60:352-360.

Bukalo O, Pinard CR, Silverstein S, Brehm C, Hartley ND, Whittle N, Colacicco G, Busch E, Patel S, Singewald N, Holmes A (2015) Prefrontal inputs to the amygdala instruct fear extinction memory formation. Sci Adv 1:1-8.

Burgos-Robles A, Vidal-Gonzalez I, Santini E, Quirk GJ (2007) Consolidation of fear extinction requires NMDA receptor-dependent bursting in the ventromedial prefrontal cortex. Neuron 53:871-880.

Cho JH, Deisseroth K, Bolshakov VY (2013) Synaptic encoding of fear extinction in mPFC-amygdala circuits. Neuron 80:1491-1507.

Cohen J (1988) Statistical power. In: Statistical power analysis for the behavioral sciences. New York, NY: Academic Press.

Criado-Marrero $M$, Morales Silva RJ, Velazquez $B$, Hernández $A$, Colon M, Cruz E, Soler-Cedeño O, Porter JT (2017) Dynamic expression of FKBP5 in the medial prefrontal cortex regulates resiliency to conditioned fear. Learn Mem 24:145-152.

Do-Monte FH, Manzano-Nieves G, Quiñones-Laracuente K, RamosMedina L, Quirk GJ (2015) Revisiting the role of infralimbic cortex in fear extinction with optogenetics. J Neurosci 35:3607-3615.

Garfinkel SN, Abelson JL, King AP, Sripada RK, Wang X, Gaines LM, Liberzon I (2014) Impaired contextual modulation of memories in PTSD: an fMRI and psychophysiological study of extinction retention and fear renewal. J Neurosci 34:13435-13443.

Grienberger C, Chen X, Konnerth A (2014) NMDA receptordependent multidendrite $\mathrm{Ca} 2+$ spikes required for hippocampal burst firing in vivo. Neuron 81:1274-1281.

Gruene TM, Roberts E, Thomas V, Ronzio A, Shansky RM (2015) Sex-specific neuroanatomical correlates of fear expression in prefrontal-amygdala circuits. Biol Psychiatry 78:186-193.

Hoover WB, Vertes RP (2007) Anatomical analysis of afferent projections to the medial prefrontal cortex in the rat. Brain Struct Funct 212:149-179.

Hunt DL, Castillo PE (2012) Synaptic plasticity of NMDA receptors: mechanisms and functional implications. Curr Opin Neurobiol 22: 496-508.

Kalisch R, Korenfeld E, Stephan KE, Weiskopf N, Seymour B, Dolan RJ (2006) Context-dependent human extinction memory is mediated by a ventromedial prefrontal and hippocampal network. J Neurosci 26:9503-9511.

Kim WB, Cho JH (2017) Synaptic targeting of double-projecting ventral CA1 hippocampal neurons to the medial prefrontal cortex and basal amygdala. J Neurosci 37:4868-4882.

Kuzmiski JB, Marty V, Baimoukhametova DV, Bains JS (2010) Stress-induced priming of glutamate synapses unmasks associative short-term plasticity. Nat Neurosci 13:1257-1264.

Kwon H-B, Castillo PE (2008) Long-term potentiation selectively expressed by NMDA receptors at hippocampal mossy fiber synapses. Neuron 57:108-120.

Langton JM, Richardson R (2009) The role of context in the reextinction of learned fear. Neurobiol Learn Mem 92:496-503.

Lenhard W, Lenhard A (2016) Computation on Effect Sizes. Dettelbach Psychom. Dettelbach, Germany: Psychometrica.

Marek R, Jin J, Goode TD, Giustino TF, Wang Q, Acca GM, Holehonnur R, Ploski JE, Fitzgerald PJ, Lynagh T, Lynch JW, Maren S, Sah P (2018) Hippocampus-driven feed-forward inhibition of the prefrontal cortex mediates relapse of extinguished fear. Nat Neurosci 21:384-392.

Maren S, Phan KL, Liberzon I (2013) The contextual brain: implications for fear conditioning, extinction and psychopathology. Nat Rev Neurosci 14:417-428.

Milad MR, Quirk GJ (2002) Neurons in medial prefrontal cortex signal memory for fear extinction. Nature 420:70-74.

Milad MR, Vidal-Gonzalez I, Quirk GJ (2004) Electrical stimulation of medial prefrontal cortex reduces conditioned fear in a temporally specific manner. Behav Neurosci 118:389-394.

Milad MR, Wright Cl, Orr SP, Pitman RK, Quirk GJ, Rauch SL (2007) Recall of fear extinction in humans activates the ventromedial prefrontal cortex and hippocampus in concert. Biol Psychiatry 62:446-454.

Milad MR, Pitman RK, Ellis CB, Gold AL, Shin LM, Lasko NB, Zeidan MA, Handwerger K, Orr SP, Rauch SL (2009) Neurobiological basis of failure to recall extinction memory in posttraumatic stress disorder. Biol Psychiatry 66:1075-1082.

Moscarello JM, LeDoux JE (2013) Active avoidance learning requires prefrontal suppression of amygdala-mediated defensive reactions. J Neurosci 33:3815-3823.

Orsini CA, Kim JH, Knapska E, Maren S (2011) Hippocampal and prefrontal projections to the basal amygdala mediate contextual regulation of fear after extinction. J Neurosci 31:17269-17277.

Orsini CA, Yan C, Maren S (2013) Ensemble coding of contextdependent fear memory in the amygdala. Front Behav Neurosci 7:199.

Padilla-Coreano N, Bolkan SS, Pierce GM, Blackman DR, Hardin WD, Garcia-Garcia AL, Spellman TJ, Gordon JA (2016) Direct ventral hippocampal-prefrontal input is required for anxiety-related neural activity and behavior. Neuron 89:857-866

Pattwell SS, Duhoux S, Hartley CA, Johnson DC, Jing D, Elliott MD, Ruberry EJ, Powers A, Mehta N, Yang RR, Soliman F, Glatt CE, Casey BJ, Ninan I, Lee FS (2012) Altered fear learning across development in both mouse and human. Proc Natl Acad Sci USA 109:16318-16323.

Peters J, Dieppa-Perea LM, Melendez LM, Quirk GJ (2010) Induction of fear extinction with hippocampal-infralimbic BDNF. Science 328:1288-1290.

Polsky A, Mel B, Schiller J (2009) Encoding and decoding bursts by NMDA spikes in basal dendrites of layer 5 pyramidal neurons. $J$ Neurosci 29:11891-11903.

Quirk GJ, Likhtik E, Pelletier JG, Paré D (2003) Stimulation of medial prefrontal cortex decreases the responsiveness of central amygdala output neurons. J Neurosci 23:8800-8807.

Rebola N, Lujan R, Cunha RA, Mulle C (2008) Adenosine A2A receptors are essential for long-term potentiation of NMDA-EPSCs at hippocampal mossy fiber synapses. Neuron 57:121-134.

Rebola N, Srikumar BN, Mulle C (2010) Activity-dependent synaptic plasticity of NMDA receptors. J Physiol 588:93-99.

Rosas-Vidal LE, Do-Monte FH, Sotres-Bayon F, Quirk GJ (2014) Hippocampal-prefrontal BDNF and memory for fear extinction. Neuropsychopharmacology 39:2161-2169.

Rougemont-Bücking A, Linnman C, Zeffiro TA, Zeidan MA, LebronMilad K, Rodriguez-Romaguera J, Rauch SL, Pitman RK, Milad MR (2011) Altered processing of contextual information during fear extinction in PTSD: an fMRI study. CNS Neurosci Ther 17:227236.

Santini E, Quirk GJ, Porter JT (2008) Fear conditioning and extinction differentially modify the intrinsic excitability of infralimbic neurons. J Neurosci 28:4028-4036.

Sepulveda-Orengo MT, Lopez AV, Soler-Cedeño O, Porter JT (2013) Fear extinction induces mGluR5-mediated synaptic and intrinsic plasticity in infralimbic neurons. J Neurosci 33:7184-7193.

Sierra-Mercado D, Padilla-Coreano N, Quirk GJ (2011) Dissociable roles of prelimbic and infralimbic cortices, ventral hippocampus, and basolateral amygdala in the expression and extinction of conditioned fear. Neuropsychopharmacology 36:529-538. 
Soler-Cedeño O, Cruz E, Criado-Marrero M, Porter JT (2016) Contextual fear conditioning depresses infralimbic excitability. Neurobiol Learn Mem 130:77-82.

Ting JT, Daigle TL, Chen Q, Feng G (2014) Acute brain slice methods for adult and aging animals: application of targeted patch clamp analysis and optogenetics. Methods Mol Biol 1183:221-242.

Wang Q, Wang Q, Song X-L, Jiang Q, Wu YJ, Li Y, Yuan TF, Zhang S, Xu NJ, Zhu MX, Li WG, Xu TL (2018) Fear extinction requires ASIC1a-dependent regulation of hippocampal-prefrontal correlates. Sci Adv 4:eaau3075.
Yizhar O, Fenno LE, Prigge M, Schneider F, Davidson TJ, O'Shea DJ, Sohal VS, Goshen I, Finkelstein J, Paz JT, Stehfest K, Fudim R, Ramakrishnan C, Huguenard JR, Hegemann P, Deisseroth K (2011) Neocortical excitation/inhibition balance in information processing and social dysfunction. Nature 477:171178.

Zinebi F, Xie J, Liu J, Russell RT, Gallagher JP, McKernan MG, Shinnick-Gallagher P (2003) NMDA currents and receptor protein are downregulated in the amygdala during maintenance of fear memory. J Neurosci 23:10283-10291. 